\title{
The effects of rate and amount of reinforcement on the speed of the pacemaker in pigeons' timing behavior
}

\author{
DAVID MACEWEN \\ Mary Washington College, Fredericksburg, Virginia \\ and \\ PETER KILLEEN \\ Arizona State University, Tempe, Arizond
}

\begin{abstract}
The peak-time procedure was used with pigeons to explore assumptions of two models of time perception: scalar expectancy theory (SET) and behavioral theory of timing (BeT). Conditions 1A and $1 \mathrm{~B}$ varied fixed-interval duration to change rate of reinforcement. Condition 2 varied hopper duration in order to manipulate arousal level. Condition 3 held constant the interval to be timed (fixed-interval duration) but varied rate of reinforcement by interspersing trials in which only the chamber light came on for a duration equal to the fixed interval. Results from Conditions 1A, $1 \mathrm{~B}$, and 3 show that the speed of the pacemaker $(1 / \tau)$ was directly proportional to the rate of reinforcement, thus supporting BeT. Manipulations of the hopper duration had no effect on the timing process.
\end{abstract}

Recent investigations in animal psychophysics have shown that animals are extremely accurate at temporal discrimination (e.g., Church \& Deluty, 1977; Dreyfus, Fetterman, Smith, \& Stubbs, 1988; Fetterman \& Dreyfus, 1987; Platt \& Davis, 1983). Given that organisms can judge the passage of time, the question remains as to how they do it. One of the first models of time perception to be developed extensively was that of Treisman (1963). His model, developed for human time perception, postulated an internal clock in which a pacemaker emits pulses at some constant rate, and those pulses increment a counter. This count is then transferred to a store from which it may be retrieved by a comparator. If two durations are to be compared, the stored count of the first duration is compared with the current count of the second.

The internal clock model has been adapted to animals' time perception by Church, Gibbon, and associates (e.g., Church, 1984; Gibbon, Church, \& Meck, 1984), who have marshalled much empirical support for their account of the timing process. Gibbon (1977) has offered an elegant set of mathematical models of temporal control, which he calls scalar expectancy theory (SET). SET assumes that animals form an expectancy of time to reinforcement and that responding is controlled by the ratio of the instantaneous (moment-to-moment) estimation to an overall estimation of time to reinforcement. If the time interval is changed, animals rescale their unit in accor-

This research was supported by National Institute of Mental Health Grant R01 MH43233 to Peter Killeen. We thank J. G. Fetterman for helpful comments on an earlier version of this manuscript. Requests for reprints should be addressed to D. MacEwen, Department of Psychology, Mary Washington College, Fredericksburg, VA 22401. dance with Weber's law. Time estimates are assumed to be distributed as a Gaussian function whose variance should increase as the square of the mean.

Killeen and Fetterman (1988) have proposed an alternative approach, which they call the behavioral theory of timing (Bet). This theory holds that time judgments are based on animals' adjunctive behaviors, which are assumed to serve as discriminative stimuli for the passage of time. The transitions between different adjunctive behaviors (e.g., from general activity to "terminal" keypecking) are precipitated by pulses from a pacemaker. Each class of behavior may be viewed as a manifestation of an underlying state, with each pulse moving the system from one state to the next. Because the pulses are assumed to occur with constant probability, transitions between states comprise a Poisson process (Killeen, Hanson, \& Osborne, 1978). The equation that embodies this process is

$$
p[N(t)=n]=(t / \tau)^{n} e^{-t / \tau} n !
$$

where $p[N(t)=n]$ is the probability that the number of pulses registered by time $t$ equals $n$ and $\tau$ is the average time between pulses (see, e.g., Killeen, in press). A key assumption of the model is that the rate of state transition (i.e., the rate of the pacemaker, $1 / \tau$ ) is directly proportional to the rate of reinforcement in the experimental context. For example, when the rate of reinforcement is doubled the rate of the pacemaker is assumed to double. This assumption is a departure from the previous internal clock models that assume the rate of the clock to be constant or, at least, not to change in a systematic way. Moreover, it provides for a possible empirical test between the BeT and SET models of time perception. 
Both models have addressed the behavior of animals under time-based schedules, particularly fixed-interval (FI) schedules with the peak procedure (Catania, 1970; Roberts, 1981). In the peak procedure, the usual FI contingencies are in effect, with the exception that, on some trials, food is omitted and the animal is allowed to respond beyond the time when food would normally be delivered. When response rates are plotted for successive segments of the food omission trials, the resulting function is approximately normal, with the peak (mode) at about the point when food would normally be delivered. Both models view this function to be the result of a timing process but assume quite different things about it.

BeT assumes that the animal advances through several interim behavioral states according to a Poisson process until it enters a terminal state of, say, keypecking. The normal distribution of pecking is the result of averaging many terminal state entries and exits. The FI scallop is the left limb of the normal distribution reflecting entries into the pecking state. The right limb, reflecting exits from the pecking state, is measurable on trials during which food is omitted. The rate at which the animal advances to the terminal state and the amount of time it resides in that state is assumed to be determined by the rate of reinforcement. The assumption of an underlying Poisson process results in a mean of $(n+1) \tau$ and a variance of $(n+1) \tau^{2}$ for the distribution in the simplest case (i.e., the animal stays in each state until one additional pulse is registered).

SET assumes that the response-rate function reflects the animal's estimates of the FI as an expectancy of time to reinforcement. These estimates are assumed to be distributed as the difference of two Gaussian distribution functions. (Roberts, 1981, fit his data to a Gaussian distribution, but SET specifies the difference of two cumulative Gaussian distributions; see Equation 2 below.) The animal begins responding only when the ratio of the local or momentary expected time to reinforcement to the overall expected time to reinforcement exceeds some threshold value (Gibbon, 1977). Both the mean and the standard deviation of the Gaussian functions are assumed to be proportional to the interval being timed; hence, the coefficient of variation $(\sigma / \mu)$ should remain constant when the FI duration is changed. The invariance of this parameter is taken as a measure of sensitivity to time comparable to the Weber fraction.

The present experiment utilized the peak procedure to assess some of the assumptions about the timing process made by BeT and to compare the results with assumptions from SET. The first manipulation varied FI duration as a way of changing rate of reinforcement. BeT assumes that the response-rate function should be described by a Poisson process and the rate of the pacemaker $(1 / \tau)$ should be directly proportional to rate of reinforcement. That is, the ratio of the values of $\tau$ at two FI durations should equal the ratio of the values of the time to reinforcement for those same two FI durations.
A second manipulation examined the possible role of arousal on $\tau$. Killeen, Hanson, and Osborne (1978) demonstrated that a single presentation of food led to an increase in arousal level as measured by general activity. They reported that different hopper durations led to different levels of general activity, but that, in all cases, activity decreased over time at the same rate. It is possible that the rate of the pacemaker is a direct function of arousal level and that changing rate of reinforcement is just one way of changing arousal. Alternatively, both the rate of the pacemaker and the arousal level are functions of rate of reinforcement, and other variables (e.g., amount) may affect one but not the other. Although SET has a motivational parameter, $H$, that is assumed to vary with the amount of (or access to) the reinforcer, $H$ gets canceled out of the expectancy ratio. SET therefore predicts that varying arousal or motivational level should have no effect on the timing process. In an attempt to change arousal level and measure the effect on the rate of the pacemaker, amount of food (hopper duration) was varied.

A third manipulation extended our investigation of the role of reinforcement rate on $\tau$. When the rate of reinforcement is decreased by doubling FI duration, the interval to be estimated is doubled, and the expectation of time to reinforcement should also double. SET and BeT make the same predictions in this case. Rate of reinforcement was therefore manipulated by holding FI duration (the interval to be estimated) constant but instituting blank trials between regular trials. These blank trials omitted keylight and reinforcement and proportionally increased the interreinforcement interval. Since the keylight was off and no food was ever presented during chamber-light-only presentations, SET would presumably hold that the timing process was not operative and no expectancy was present. Thus, $\tau$ could be manipulated while holding constant expectation to reinforcement. Because expectancy of time to reinforcement does not change in this manipulation, SET predicts no changes in parameter values in its equations. Under the two different rates of reinforcement (chamber-light condition vs. no-chamber-light condition), BeT predicts that the value of $\tau$ should vary and the ratio of the values of $\tau$ should equal the ratio of the values of the two different interreinforcement intervals.

\section{Method}

\section{Subjects}

Four Silver King pigeons were maintained at $80 \%( \pm 10 \mathrm{~g})$ of their free-feeding weights. All animals had previous experience keypecking for food under various schedules of reinforcement.

\section{Apparatus}

A standard two-key Lehigh Valley Electronics pigeon chamber served as the experimental space. The left key remained dark and inoperative throughout the experiment. The right key was $22 \mathrm{~cm}$ above the chamber floor and could be illuminated by a green light. Key switch closure required a force of 0 . 1N. Chamber illumination was provided by a miniature lamp centered on the front wall $32 \mathrm{~cm}$ above the floor. Mixed grain was occasionally presented 
through an opening centered on the front wall, $5 \mathrm{~cm}$ square and $10 \mathrm{~cm}$ above the chamber floor. White noise was continuously presented to mask extraneous sounds. A ventilation fan provided fresh air and additional masking. A microcomputer controlled the experiment and recorded data.

\section{Procedure}

All birds were exposed to an autoshaping program for two sessions and then switched to FI schedules of reinforcement. The FI duration was increased over the next few sessions until all birds were responding under a FI 30-sec requirement. During the fixed interval, the key was illuminated with a green light and the chamber was illuminated by the white chamber light. A key switch closure following the FI $30 \mathrm{sec}$ turned off the keylight and the chamber light and provided 3-sec access to mixed grain. The 3-sec feed duration was timed from the point when the bird broke a photobeam with its head upon entering the feeder opening. Following food presentation, all lights remained off for a 15-sec intertrial interval (ITI). This sequence of events was repeated for 60 grain presentations (trials). All subjects showed stable responding under the FI 30sec schedule after 10 consecutive days. Stability was assessed by visual inspection of a plot of the latencies from keylight onset until the third keypeck.

After the 10th session, 2 subjects were switched to FI 14-sec (Birds 14 and 41), 2 subjects were switched to FI 35-sec (Birds 36 and 37), and a peak procedure was instituted. For 12 of the 60 trials, the contingency between a keypeck and grain delivery was not in effect; instead, the trial continued for twice the usual FI duration. Thereafter, the trial terminated with a probability of 0.15 every $2 \mathrm{sec}$ (for the FI $14 \mathrm{sec}$ ) or every $5 \mathrm{sec}$ (for the FI $35 \mathrm{sec}$ ). This continued for a maximum of six possible additional extensions. Whenever the trial was terminated, no grain was delivered and all lights went off for the 15-sec ITI. If the FI is $x$, with bin size of $x / 7$, the average duration of a peak trial is $3 x$. The 12 peak trials were randomly presented each session.

\section{Condition 1A}

Two birds (Birds 14 and 41) were trained under a peak procedure using an FI 14-sec schedule; the other 2 birds (Birds 36 and 37 ) were similarly trained using an FI $35-\mathrm{sec}$ schedule. The criterion for stable responding was the same as mentioned above. All birds were trained for a minimum of 25 days.

\section{Condition 2}

Duration of food was varied. Birds 14 and 37 were switched to 1.5-sec food access and Birds 41 and 36 were switched to 7-sec food access. After 25 days, food-access times were reversed: Birds 14 and 37 received 7-sec access to food and Birds 41 and 36 received 1.5 -sec access to food for an additional 25 days.

\section{Condition 1B}

All birds were returned to conditions with 3-sec access to food for 10 sessions. The FI durations were then switched for the birds. Birds 14 and 41 were switched to an FI 35-sec schedule; Birds 36 and 37 were switched to an FI 14-sec schedule. All birds continued to receive 12 randomly presented peak trials each session. All birds showed stable responding after 25 daily sessions.

\section{Condition 3}

The contingencies of Condition 1B remained in effect during Condition 3 , except that the chamber light came on for a duration equal to the FI after each ITI. During this time, the keylight was off, the key switch was inoperative, and no food was ever presented. Following the offset of the chamber light, a 15-sec ITI was again in effect. Peak and regular FI trials alternated with chamber-lightonly exposures throughout the session. These contingencies were maintained for 25 days. Birds 14 and 41 were then switched to an
FI 14-sec schedule and birds 36 and 37 were switched to an FI 35sec schedule, with the chamber-light manipulation still in effect. These contingencies were again maintained for 25 consecutive days.

\section{Dependent Measures}

Rates of keypecking during peak trials were recorded for each of the 20 possible response bins. The bimeans (see Killeen, 1985) over the last 10 days of each experimental phase were obtained for each response bin. In addition, latencies to the third keypeck for each trial were collected for each session in each phase. No data were recorded during ITI, food presentations, or chamber-lightonly trials. Only data from peak trials were used in the analysis.

\section{Inferred Measures}

The parameters $\mu$ and $\sigma$ were inferred by fitting Equations 2 and 5 to the data choosing values that minimized the sum of squared deviations. The parameters $\tau$ and $n$ were then inferred from $\mu$ and $\sigma$ by solving Equations 3 and 4 . In all cases, parameters were derived from averaged data of the subjects that experienced the same contingencies.

\section{RESULTS}

\section{Condition 1}

Figure 1 presents response rates for each of the first 13 bins during peak trials of Conditions $1 \mathrm{~A}$ and 1B. Data points (squares) represent the bimean of response rates for the last 10 sessions averaged over the 4 birds. The top panel shows data from the FI 14-sec condition $(t=$ $14 \mathrm{sec})$; the bottom panel shows data from the FI 35-sec condition $(t=35 \mathrm{sec})$. In both cases, the data increase
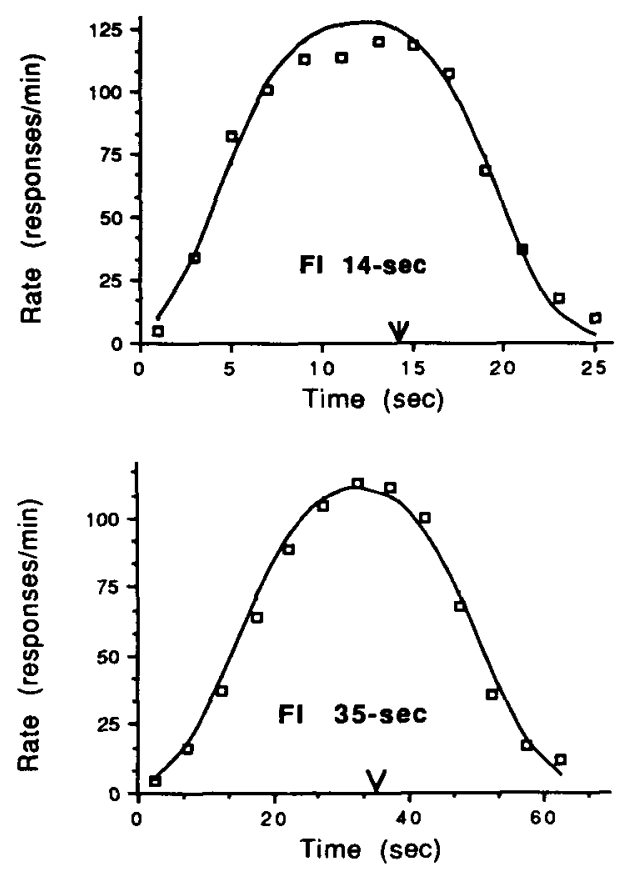

Figure 1. Response rates (pecks per minute) for each of the first 13 bins during peak trials in Conditions $1 \mathrm{~A}$ and 1B. Each data point represents the bimean of response rates for the last 10 sessions averaged over the 4 birds. The curves are derived from Equations 2 and 5 . 
smoothly to a maximum close to the expected time of reinforcement and then decrease to a minimum at twice the training value. Thereafter, the data for the remaining bins (not shown) increase erratically to about one fourth of the peak rate. Church, Miller, Gibbon, and Meck (1988) reported a similar delayed increase for rats and, in a careful series of experiments, demonstrated that it disappeared when several factors confounding the basic timing task were minimized.

The theoretical response rate $\left(\boldsymbol{R}_{t}\right)$ is proportional to the difference of two normal distributions:

$$
R_{t}=k\left[\phi\left(\mu_{1}, \sigma_{1}\right)-\phi\left(\mu_{2}, \sigma_{2}\right)\right],
$$

where $\phi(\mu, \sigma)$ is a cumulative normal distribution of mean $\mu$ and standard deviation $\sigma$, and $k$ is a constant of proportionality.

Both SET and BeT employ this equation to account for various types of data (Gibbon, 1977). BeT assumes that the underlying system is a Poisson process-an assumption that we treat as a "default" assumption, because it provides one of the more obvious and tractable models. A more reasonable assumption might be that the pacemaker is somewhat more accurate and the accumulator is somewhat less than perfect. This would also lead to Equation 2 as a convenient approximation, but would stipulate different relationships among the parameters of the distributions (see the Appendix of Killeen \& Fetterman, 1988).

Under the Poisson assumption, the mean and variance of the normal distribution governing entry into the response state are

$$
\mu=(n+1) \tau
$$

and

$$
\sigma^{2}=(n+1) \tau^{2},
$$

where $n$ is the number of pulses from the pacemaker before pecking begins and $\tau$ is the period of the pacemaker. The mean and variance of the distribution governing exit from the response state are governed by similar equations. A theoretical assumption reduces the number of free parameters: According to BeT, $\tau$ must remain invariant within the experimental context, and so the calculations for both entry and exit share the same values of $\tau$.

How long will the animals stay in the response state? That is an empirical question, and the data, as viewed through this model, permit anywhere between one and three pulses before the animal exits from the response state. However, changes in the assumed dwell time had very little effect on the goodness of fit; so, for simplicity, we have assumed that residence time is uniformly a single pulse and have analyzed all the data according to that convention. Thus, in calculating the parameters of the normal distributions for exit, we use $n+1$ and $\tau$.

Equation 2 gives the probability of being in the response state correlated with pecking, but it does not tell us the response rate while in that state; it is not immediately obvious how to translate probability into rate. The problem arises because responses are not instantaneous. Each time a response is made, it reduces the time available during which other responses might be emitted. If responses are attempted randomly in time at a "theoretical" rate of $R_{t}$, and each response blocks the emission of other responses for $(\delta)$ seconds, then the measured response rate $\left(R_{m}\right)$ will be approximately

$$
R_{m}=R_{t} /\left(1+\delta R_{t}\right)
$$

(Bharucha-Reid, 1960, Equation 51; Killeen, 1981, Equation 19). At low rates of responding and for small values of $\delta, R_{m}=R_{t}$; however, at response rates that are high relative to the maximum rate, this ceiling effect will cause a concave departure from linearity toward an asymptotic rate of $1 / \delta$.

The above mapping was used to get from the probabilistic predictions of Equation 2 to actual response rates. The equations introduce two new parameters: the rate of responding given that the animal is in the response state correlated with pecking $\left(R_{m}\right)$ and the maximum sustainable response rate $(1 / \delta)$. Although a model such as this was necessary to accommodate these data, the fit is not perfect (as can be seen in the top panel of Figures 1 and 4), where the rates came against a firmer ceiling than that pictured by Equation 5 .

Values for the key parameters from Equations 2-5 are presented in Table 1 for the two FI values of Conditions $1 \mathrm{~A}$ and $1 \mathrm{~B}$. In addition, Table 1 shows the proportion of variance accounted for by the model. Figure 2 presents the recovered values of $\tau$ across reinforcement conditions for each bird (Birds 14, 41, 36, and 37), as well as a function fit to the averaged data.

A key assumption in BeT is that the value of $\tau$, the period of the pacemaker, should be proportional to the time between reinforcements. All birds showed an increase in the value of $\tau$ when the period of reinforcement was decreased by shifting from FI $14 \mathrm{sec}$ to FI $35 \mathrm{sec}$. The slope of the line fit to the averaged data was reliably greater than zero $[t(3)=8.09, p<.01]$. Under this peak procedure, the ratio of values for the average time between reinforcers under the two conditions was 2.5 and the ratio of the two FI values was also 2.5. The ratio of $\tau$ under FI 35 to $\tau$ under FI 14 was found to be 2.7, close to the predicted value of 2.5. The slope of the regression line fit to values of $\tau$ under each FI condition was not reliably different from $2.5[t(3)=1.55$, n.s.], supporting the notion that $\tau$ is directly proportional to the time between

\section{Table 1}

Key Parameters of Equations 2-5 and Proportion of Variance Accounted for by the Model for Conditions $1 A$ and $1 B$

\begin{tabular}{cccccr}
\hline Subject & $n$ & $\tau(14 \mathrm{sec})$ & $\tau(35 \mathrm{sec})$ & $\delta$ (msec) & PVA \\
\hline 14 & 8 & 1.4 & 3.6 & 375 & .972 \\
41 & 8 & 1.3 & 3.5 & 240 & .985 \\
36 & 7 & 1.6 & 4.3 & 480 & .916 \\
37 & 6 & 1.6 & 4.4 & 480 & .955 \\
Average & 7 & 1.5 & 4.1 & 375 & .957 \\
\hline
\end{tabular}

Note-PVA $=$ proportion of variance that was accounted for by the model. 


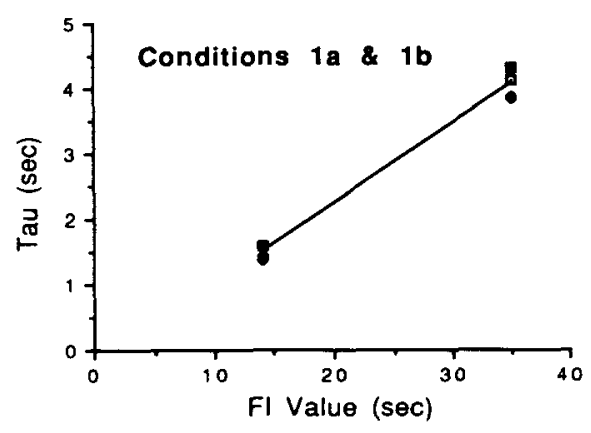

Figure 2. The recovered values of $\tau$ across reinforcement conditions of FI $14 \mathrm{sec}$ and FI 35 sec for each of the 4 birds (Birds 14, 41, 36, and 37) from Conditions $1 A$ and $1 B$. The regression line through the average data shows that $r$ is proportional to the time between reinforcements.

Table 2

Key Parameters of Equations 2-5 and Proportion of Variance Accounted for by the Model for Condition 2

\begin{tabular}{ccccc}
\hline Amount & $n$ & $\tau(\mathrm{sec})$ & $\delta(\mathrm{msec})$ & PVA \\
\hline \multicolumn{5}{c}{ FI 14 sec } \\
1.5 & 7 & 1.5 & 375 & .964 \\
3.0 & 7 & 1.5 & 375 & .972 \\
7.0 & 7 & 1.6 & 375 & .964 \\
& & FI $35 \mathrm{sec}$ & & \\
1.5 & 7 & 3.9 & 315 & .962 \\
3.0 & 7 & 4.1 & 315 & .971 \\
7.0 & 7 & 3.9 & 315 & .989 \\
\hline
\end{tabular}

Note-PVA $=$ proportion of variance that was accounted for by the model.

reinforcements. The use of a fixed intertrial interval might have made it possible for the pigeons to ignore the stimuli and time each trial from the end of the previous trial. That this did not happen is shown by the data in Figure 1, which rise from an origin of $0 \mathrm{sec}$ at the beginning of the interval. The count started when the keylight came on. It is nonetheless possible that the ITI added to the timebase for reinforcement and thus affected the speed of the pacemaker. Because we did not differentially manipulate ITI in this experiment, however, we cannot comment on this possibility.

\section{Condition 2}

Table 2 presents key parameters from Equations 2-5 for each of the two FI durations at each hopper duration $(1.7,3.0$, and $7.0 \mathrm{sec})$, as well as the proportion of variance accounted for by the model. The data for the FI 14sec schedule are averaged over Birds 14 and 41 , and those for the FI 35-sec schedule are averaged over Birds 36 and 37. Data for the 3.0-sec hopper duration were taken from Condition $1 \mathrm{~A}$ and averaged over all 4 subjects.

Figure 3 shows the values of $\tau$, the period of the pacemaker, when amount of reinforcement was varied. The solid bars correspond to the FI 14-sec condition; the stippled bars correspond to the FI 35-sec condition. Food amount (hopper duration) had no effect on $\tau$. Peak response rates for the 1.5-, 3.0-, and 7.0-sec durations were 144,122 , and 131 responses per minute for the FI 14-sec condition and 101, 108, and 89 responses per minute for the FI 35-sec condition.

\section{Condition 3}

Table 3 presents key parameters of Equations 2-5 under the standard peak procedure described earlier and when sessions were extended with trials in which the chamber light was kept on for a duration equal to the FI schedule in effect. The intent of this manipulation was to decrease the period of reinforcement while holding the duration to be timed constant. The data for the FI 14-sec schedule are averaged over Birds 41 and 37, and those for the FI 35-sec schedule are averaged over Birds 14 and 36. Proportion of variance accounted for by the model is also presented in Table 3. The period of the pacemaker was clearly decreased by the extended time between reinforcements. Table 3 shows that when the values of $\tau$ were held at those obtained from the standard peak procedure of Conditions 1A and 1B, proportion of variance accounted for decreased considerably (even though $n$ was let to float to the value that optimized goodness of fit). We cannot presume constancy of the pacemaker without giving up 10 points of accuracy in fitting the data. Letting $\tau$ assume the value that maximizes goodness of fit, Table 3 shows that, for the FI 14-sec schedule, $\tau$ decreased from $1.5 \mathrm{sec}$ in the standard condition to $2.4 \mathrm{sec}$ in the extended condition; for the FI 35-sec schedule, $\tau$ decreased from $4.0 \mathrm{sec}$ in the standard condition to $6.8 \mathrm{sec}$ in the extended condition.

The average time ( $T$ ) between reinforcers was proportional to the FI value; the ratio of the values of $T$ under the standard and chamber-light-extended conditions was 1.71. The slope of the regression of the data was reliably greater than zero $[t(3)=4.63, p<.01]$ and not reliably different from $1.71[t(3)=.67$, n.s.]. Thus, the hypothesis that the period of the pacemaker is directly proportional to the time between reinforcements was again supported.

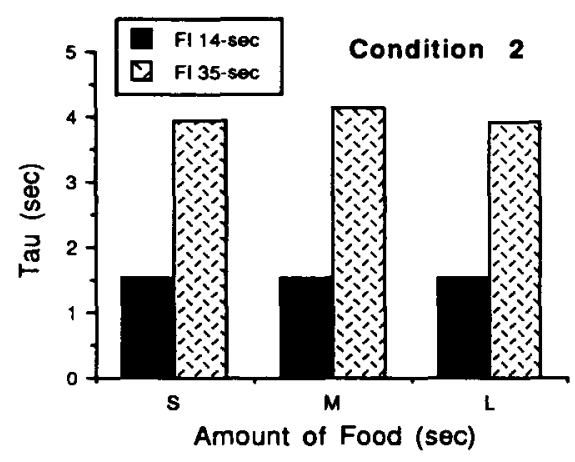

Figure 3. Bars represent average values of $r$ when amount of reinforcement (hopper duration) was varied. Solid bars represent the FI 14-sec schedule; stippled bars represent the FI 35-sec schedule. 
Table 3

Key Parameters of Equations 2-5 and Proportion of Variance Accounted for by the Model for Condition 3

\begin{tabular}{lllll}
\hline Condition & $n$ & $T(\mathrm{sec})$ & $\delta(\mathrm{msec})$ & PVA \\
\hline & \multicolumn{5}{c}{ FI 14 sec } & & \\
Standard & 7 & 1.5 & 315 & .977 \\
Extended & 7 & $1.5^{*}$ & 315 & .819 \\
Extended & 4 & 2.4 & 315 & .942 \\
& & FI $35 \mathrm{sec}$ & & \\
Standard & 7 & 4.0 & 375 & .984 \\
Extended & 6 & $4.0^{*}$ & 375 & .838 \\
Extended & 3 & 6.8 & 375 & .931 \\
\hline
\end{tabular}

Note-PVA = proportion of variance that was accounted for by the model. *Values were held at the standard value and the fit was otherwise optimized.

Figure 4 shows response rates (pecks per minute) for each of the first 13 bins during peak trials of Conditions $1 \mathrm{~A}$ and $1 B$ (filled squares) and Condition 3 (open squares). The top panel shows the data from the FI 14-sec schedule; the bottom panel shows the data from the FI 35-sec schedule. Data points are bimeans of response rates for the last 10 sessions averaged over the 2 birds in each condition. As before, the fitted curves are derived from the difference between two normal distributions. Although in both cases
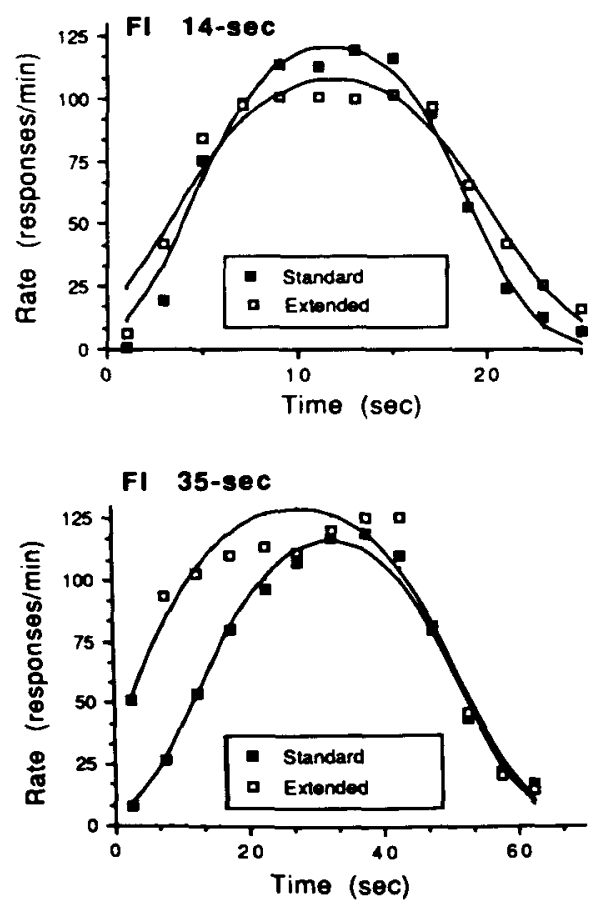

Figure 4. Response rates (pecks per minute) for each of the first 13 bins during peak trials of Condition $1 A$ and $1 B$ (filled squares) and for peak trials of Condition 3 where interreinforcement time was extended (open squares). The top panel shows the data from the FI 14-sec schedule; the bottom panel shows the data from the FI 35-sec schedule. Data points are bimeans of response rates for the last 10 sessions averaged over the 2 birds in each condition. Smooth curves are functions fit to the data from Equations 2 and 5. the functions approximate the data, the approximation is better for the peak trials of Conditions $1 \mathrm{~A}$ and $1 \mathrm{~B}$ than for peak trials from the extended interreinforcement duration of Condition 3. The variance of the distribution increased under this manipulation, as expected, although the mean of the distributions either did not change (FI $14 \mathrm{sec}$ ) or decreased. Equations 3 and 4 show us that changes in $\tau$ can be compensated for by changes in $n$ to keep either $\mu$ or $\sigma^{2}$ constant, but no compensatory changes can keep both constant. Under the FI 14-sec schedule, the pigeons kept $\mu$ constant at the price of an increase in $\sigma^{2}$. Under the FI 35-sec schedule, $\sigma^{2}$ increased and $\mu$ decreased.

\section{DISCUSSION}

Conditions $1 \mathrm{~A}$ and $1 \mathrm{~B}$ replicated the "peak-time" effect in pigeons and showed that the scalar timing demonstrated was consistent with predicted variations in the speed of the pacemaker. The period of the pacemaker $(1 / \tau)$ was found to be directly proportional to the period of reinforcement in the experimental setting. By themselves, however, the results of Conditions $1 \mathrm{~A}$ and $1 \mathrm{~B}$ do not differentiate between the assumptions of SET and BeT. Because the period of reinforcement was manipulated by changes in FI schedule duration, one could argue that either the period of the pacemaker or the expectation to reinforcement was the causal factor. In Condition 2, changes in amount of reinforcement had no effect on the parameters of the distributions fit to the data. This finding is consistent with the SET model, in which the motivational parameters cancel out of the expectancy ratios underlying timing performance. We had expected to see some effect of amount in Condition 2, since changes in arousal due to footshock affect pacemaker speed (Meck, 1983). However, in a recent review of the literature concerning the effects of magnitude of reinforcement, Bonem and Crossman (1988) concluded that "the question of whether magnitude of reinforcement is or is not effective remains open to debate"' (p. 359). Some studies have even found an inverse relation between amount of reinforcement and response rate (Staddon, 1970). Staddon explains these results as being due to a postreinforcement inhibitory effect similar to that found with the omission procedure. Notice that in the present experiment there was no systematic change in peak rate as amount of food was varied-if anything, it tended to decrease for the largest amount. Although this 5-to-1 change in amount of food had no systematic effect on response rate, the 2.5-to-1 change in reinforcement rate between the 14- and the 35sec schedules did have a systematic effect on peak rate in the expected direction. Thus, there is no evidence that manipulation of amount of food affected arousal level (as inferred from response rate). It is also certainly apparent in Figure 3 that manipulation of amount of food had no effect on the speed of the pacemaker. Beyond that, it is not clear in the present experiment whether (1) arousal level does affect pacemaker speed, but difference in hopper time did not generate differences in arousal, or 
(2) arousal level does not determine pacemaker speed, even though it may be affected by some of the same variables that affect pacemaker speed.

Condition 3 showed that extending the interreinforcement interval without changing the interval to be estimated (FI duration) affected the speed of the pacemaker. These results are similar to those of Holder and S. Roberts (1985) and S. Roberts and Holder (1985), who used a peak procedure and found that stimulus durations were not timed by the rats unless the signal was directly paired with primary reinforcement. In addition, W. A. Roberts, Cheng, and Cohen (1989) found that when pigeons were tested with time-outs midway through a peak trial, they did not continue timing during the time-out, but reset the timing mechanism to $0 \mathrm{sec}$. These procedures, using both rats and pigeons, are analogous to our chamber-light-only presentations, which were never directly paired with reinforcement and were always followed by an ITI. The results of Condition 3 are consistent with the assumptions and predictions of the BeT model. Since the interval to be timed is not changed in Condition 3, the assumptions from SET predict that scalar timing should not be affected. However, we can see in Figure 4 that the variance increased and the pacemaker slowed.

Taken together, the results from all three conditions do not unequivocally rule out either model. In the standard peak procedure using FI schedules, both models make the same predictions. The results from Condition 2 follow from SET, since the motivational parameter, $H$, plays no role in the final expectancy ratio. The results clarify some of the causal factors in BeT: they teach us that amount of reinforcement may have no effect on the pacemaker speed for pigeons. However, the results do not rule out the hypothesis that pacemaker speed may be controlled by arousal level, because there is no evidence from response rate that arousal level was changed by the different amounts of food. The results of Condition 3 favor the $\mathrm{BeT}$ model. One central assumption of $\mathrm{BeT}$ is that the rate of the pacemaker is directly proportional to the rate of reinforcement. When the interval to be timed is held constant while rate of reinforcement is varied, BeT predicts that the animal's clock will also vary. SET has no means to accommodate the findings of Condition 3 , since expectation to reinforcement does not change and clock speed is assumed to not vary. Neither model, however, would take pride of ownership in the mediocre fit of Equation 2 to the data from the extended trials of Condition 3.

\section{REFERENCES}

BhaRUCHA-REID, A. T. (1960). Elements of the theory of Markov processes and their applications. New York: McGraw-Hill.

BONEM, M., \& CROSSMAN, E. K. (1988). Elucidating the effects of reinforcement magnitude. Psychological Bulletin, 104, 348-362.

Catania, A. C. (1970). Reinforcement schedules and psychophysical judgments: A study of some temporal properties of behavior. In W. N.
Schoenfeld (Ed.), The theory of reinforcement schedules (pp. 1-42). New York: Appleton-Century-Crofts.

Church, R. M. (1984). Properties of the internal clock. In J. Gibbon \& L. Allan (Eds.), Annals of the New York Academy of Sciences: Vol. 423. Timing and time perception (pp. 566-582). New York: New York Academy of Sciences.

Church, R. M., Deluty, M. Z. (1977). Bisection of temporal intervals. Joumal of Experimental Psychology: Animal Behavior Processes, 3, 216-228.

Church, R. M., Miller, K. D., Gibbon, J., \& Meck, W. (1988, November). Symmetrical and asymmetrical sources of variance in temporal generalization. Paper presented at the annual meeting of the Psychonomic Society, Chicago.

Dreyfus, L. R., Fetterman, J. G., Smith, L. D., Stubis, D. A. (1988). Journal of Experimental Psychology: Animal Behavior Processes, 14, 349-367.

Fetterman, J. G., \& Dreyfus, L. R. (1987). Duration comparison and the perception of time. In M. L. Commons, J. E. Mazur, J. A. Nevin, \& H. Rachlin (Eds.), Quantitative analysis of behavior: Vol. 5 . Reinforcer value - The effect of delay and intervening events (p. 27). Hilisdale, NJ: Erlbaum.

GiBBON, J. (1977). Scalar expectancy theory and Weber's law in animal timing. Psychological Review, 84, 279-325.

Gibbon, J., Church, R. M., Meck, W. H. (1984). Scalar timing in memory. In J. Gibbon \& L. Allan (Eds.), Annals of the New York Academy of Sciences: Vol. 423. Timing and time perception (pp. 5277). New York: Appleton-Century-Crofts.

Holder, M., RoberTs, S. (1985). Comparison of timing and classical conditioning. Journal of Experimental Psychology: Animal Behavior Processes, 11, 172-193.

KILleEN, P. R. (1981). Averaging theory. In C. M. Bradshaw, E. Szabadi, \& C. F. Lowe (Eds.), Quantification of steady-state operant behaviour (pp. 21-34). New York: Elsevier.

KilleEN, P. R. (1985). The bimean: A measure of central tendency that accommodates outliers. Behavior Research Methods, Instruments, \& Computers, 17, 526-528.

KILLEEN, P. R. (in press). Behavior's time. In G. H. Bower (Ed.), The psychology of learning and motivation: Advances in research and theory (Vol. 27).

Killeen, P. R., \& Fetterman, J. G. (1988). A behavioral theory of timing. Psychological Review, 95, 274-295.

Killeen, P. R., Hanson, S. J., \& Osborne, S. R. (1978). Arousal: Its genesis and manifestation as response rate. Psychological Review, 85, 571-581.

MEck, W. H. (1983). Selective adjustment of the speed of internal clock and memory processes. Journal of Experimental Psychology: Animal Behavior Processes, 9, 171-201.

PlatT, F. R., \& Davis, E. R. (1983). Bisection of temporal intervals by pigeons. Journal of Experimental Psychology: Animal Behavior Processes, 9, 160-170.

ROBERTS, S. (1981). Isolation of an internal clock. Journal of Experimental Psychology: Animal Behavior Processes, 7, 242-268.

Roberts, S., \& Holder, M. D. (1985). Effects of classical conditioning on an internal clock. Journal of Experimental Psychology: Animal Behavior Processes, 11, 194-214.

Roberts, W. A., Cheng, K., \& Cohen, J. S. (1989). Timing light and tone signals in pigeons. Journal of Experimental Psychology: Animal Behavior Processes, 15, 23-35.

STADDON, J. E. R. (1970). Effects of reinforcement duration on fixedinterval responding. Joumal of the Experimental Analysis of Behavior, 13, 1-31.

Treisman, M. (1963). Temporal discrimination and the indifference interval: Implications for a model of the "internal clock." Psychological Monographs, 77(Whole No. 756).

(Manuscript received August 3, 1989; revision accepted for publication December 6, 1990.) 